\title{
High Intensity Focused Ultrasound (HIFU) Treatment for Ablating of Uterine Fibroids, Experience of Saudi Arabia
}

MS Aljuhani ${ }^{1}$, M Alkhorayef ${ }^{1}$, MZ Mahmoud $^{2,3}$, MA Fagiri $^{3}$, C Saade $^{4}$, KS Alzimami $^{1}$ F Mayah $^{5}$

\begin{abstract}
Objective: This study was designed with an aim to evaluate the effectiveness of high intensity focused ultrasound (HIFU) ablation for the treatment of uterine fibroids.

Methods: Patients with symptomatic fibroids (mean age \pm SD was $(38.1 \pm 1.3)$ years; age ranges between 28 to 48 years) were undergoing HIFU ablation between January 2014 and January 2015. A HIFUNIT 9000 tumor therapy was used. Pre- and post-treatment imaging examination included B-scan imaging, color Doppler flow imaging (CDFI), and power Doppler imaging was performed and analyzed. Statistical analysis was performed using the standard Statistical Package for the Social Sciences (SPSS Inc., Chicago, IL, USA) version 15 for windows. $P \leq 0.0001$ was considered to indicate a statistical significant difference.
\end{abstract}

Results: The mean diameter of the uterine fibroids was $(5.2 \pm 1.8) \mathrm{cm}$ (diameter range between 3.9 to 10.6 $\mathrm{cm}$ ), and the mean volume was $(45.2 \pm 1.6) \mathrm{cm}^{3}$ (volume range between 7.8 to $76.4 \mathrm{~cm}^{3}$ ). HIFU ablation was well tolerated in all patients. No major complications occurred. During follow-up, the ablated fibroids shrank significantly over time. The symptoms were alleviated significantly. No patients had amenorrhoea after treatment. Vaginal expulsion of necrotic tissue was seen in $(12 ; 8 \%)$ of patients after HIFU ablation, which disappeared after 5-6 menstrual cycles.

Conclusion: Ultrasound guided HIFU ablation may be a safe and effective minimally invasive technique for the treatment of submucosal fibroids. Further studies are warranted to observe its influence on fertility.

Keywords: Color doppler flow imaging, extracorporeal, high intensity focused ultrasound, uterine artery embolization

From: ${ }^{1}$ Department of Radiological Sciences, College of Applied Medical Sciences, King Saud University, Riyadh, Saudi Arabia, ${ }^{2}$ Radiology and Medical Imaging Department, College of Applied Medical Sciences, Salman bin Abdulaziz University, Al-Kharj, Saudi Arabia, ${ }^{3}$ Basic Sciences Department, College of Medical Radiological Sciences, Sudan University of Science and Technology, Khartoum, Sudan ${ }^{4}$ Department of Diagnostic Radiology, American University of Beirut Medical Center, Beirut, Lebanon, ${ }^{5}$ Radiation Therapy Department, King Fahad Medical City, Riyadh, Saudi Arabia

Correspondence: Dr. MZ Mahmoud, Radiology and Medical Imaging Department, College of Applied Medical Sciences, Salman bin Abdulaziz University, PO Box 422, Al-Kharj 11942, Saudi Arabia.

Fax: 00966115886301; e-mail: m.alhassen@sau.edu.sa 


\section{INTRODUCTION}

Uterine fibroids are common uterine benign neoplasm worldwide. They can cause severe menstrual bleeding and pelvic discomfort $(1,2)$. In (10-20\%) of women these uterine fibroids lead to symptoms, such as heavy and prolonged menstrual bleeding, pain, urinary frequency or urgency, bulk-related symptoms, and constipation (3). Surgical intervention is the most common method of treatment if symptoms such as substantial bleeding or pelvic pain appear $(1,2)$. Myomectomy or hysterectomy is the most common method of treatment if symptoms such as substantial bleeding or pelvic pain appear. However, hysterectomy is not accepted by women who have a strong desire for a future pregnancy. Therefore, nonsurgical treatment is the only available option for those women who want their uterus preserved $(4,5)$.

Several minimally invasive techniques have been introduced providing local control of uterine fibroids and for preserving the uterine. One of these techniques, high intensity focused ultrasound (HIFU) has been widely used for the treatment of solid tumors including uterine fibroids (6,7). As a non-invasive modality, HIFU therapy is receiving increasing interest for the treatment of localized solid malignancies and benign tumors because it is capable of producing coagulative necrosis at a precise focal point within the body, without harming overlying and adjacent structures even within the path of the beam (8). Moreover, high-intensity ultrasonic beams can be focused with high accuracy using an extracorporeal transducer to thermally ablate tumors without the need to introduce needles or probes into the tumor (9).

In the past few decades, several clinical HIFU projects have been conducted by various research groups, and substantial results indicate that HIFU treatment would be safe, effective, and feasible in clinical applications $(6,10-13)$. In this article, the authors introduce our experience with using extracorporeal HIFU for the treatment of patients with uterine 
fibroids, to see the effectiveness of HIFU treatment in refilling symptoms of uterine fibroids in Saudi women treated in the HIFU Center in King Fahad Medical City (KFMC).

\section{MATERIAL AND METHODS}

\section{Selection and description of participants}

After receiving approval from the local ethics committee, patients with symptomatic fibroids were enrolled in this prospective study at in KFMC. Patients who had other pelvic or uncontrolled systematic disease were excluded, as were postmenopausal women and women who were unable to communicate with the researchers during the treatment. Women who had undergone previous abdominal surgery were examined for scars, and if these were extensive in the path of the ultrasound beam, these patients were excluded from the study. Women who had multiple fibroids were also ineligible for the study.

Finally, 150 consecutive patients with 150 uterine fibroids underwent HIFU ablation between January 2014 and January 2015. The mean age \pm SD was $(38.1 \pm 1.3)$ years (age range between 28 to 48 years). The mean diameter of the uterine fibroids was $(5.2 \pm 1.8) \mathrm{cm}$ (diameter range between 3.9 to $10.6 \mathrm{~cm}$ ), and the mean volume was $(45.2 \pm 1.6) \mathrm{cm}^{3}$ (volume range between 7.8 to $76.4 \mathrm{~cm}^{3}$ ). Patient data and characteristics of uterine fibroids were described in (Table 1).

\section{HIFU therapy system}

A HIFUNIT 9000 tumor therapy system (Shanghai Aishen Technology, China) was used. The instrument was composed of three parts: a therapeutic transducer located in a degassed water tank, an imaging system comprising an ultrasound probe coupled with a sterotaxic localizing arm, and a computer controlled system for firing energy and moving the therapeutic transducer in three dimensions. The energy is produced from a $20 \mathrm{~cm}$ diameter therapeutic transducer with a focal length of $150 \mathrm{~mm}$ operating at a frequency of $0.9 \mathrm{MHz}$. The focal region is $9.8 \mathrm{~mm}$ along the beam axis and $1.3 \mathrm{~mm}$ in the transverse direction. In the 
center of the HIFU transducer, a 3.5-5 MHz convex diagnostic ultrasound probe is mounted to provide real-time imaging for targeting.

The ultrasound coupling gel was applied to eliminate air pockets trapped between the membrane and the patient's skin. Spatial-average temporal-average intensity of the therapeutic transducers, was 0 to $3000 \mathrm{~W} / \mathrm{cm}^{2}$. The frequency of the ultrasound wave was 1.0 MHz. Focal region of the therapeutic transducers was an ellipsoid with dimensions of $8 \mathrm{~mm}$ along the beam axis and $3 \mathrm{~mm}$ in the transverse direction at the $17 \mathrm{~cm}$ focal distance from each therapeutic transducer.

\section{Pretreatment preparation and imaging work-up}

Before HIFU ablation, patients underwent careful bowel preparation, including low residue diet for 3 days, fasting for 12 hours before treatment, administration of cathartics in the evening the day before treatment, and an enema in the early morning of the treatment day. A catheter was inserted into the bladder and connected to degassed sterile water through an infusion set for the purpose of properly filling the bladder during HIFU ablation. Degassed water balloons of different size were used to compress and push away the bowel from the acoustic pathway during treatment when necessary. Pre- and post-treatment imaging examination included B-scan imaging, color Doppler flow imaging (CDFI), and power Doppler imaging was performed and analyzed.

High-resolution Hitachi hi-vision 6500 digital ultrasound system (model number: hi vision 6500) equipped with a convex probe with a frequency of 2-3.5 MHz (model 2302650, serial number 1028924YM7), manufactured in February 2010, and made by Yokogawa Medical Systems, Ltd. (7-127 Asahigaoka 4-chome, Hino-shi, Tokyo, Japan) was used.

\section{HIFU therapy procedure}

Patients were carefully placed in the prone position, with the abdominal skin in contact with the degassed water. The fibroids were divided into sections with $5 \mathrm{~mm}$ separation on 
ultrasound. HIFU energy exposures of 1-3 sec separated by 2-3 sec were applied to ablate one target spot. An acoustic power of $420-520 \mathrm{~W}$ was used in this study. If the echogenicity of the target spot did not change after firing energy, the energy exposure was repeated until the echogenicity of the target spot became increased in the ultrasound. To avoid thermal damage to adjacent structures such as bowel, bladder and endometrium, the focus of the energy beam was kept at least $1 \mathrm{~cm}$ away from the tumor margin.

By scanning the HIFU beam in successive sweeps from the deep in the shallow regions of the fibroid, the targeted regions on each slice could be completely ablated. After ablation of one spot, the transducer was moved, and a nearby spot was treated similarly. This process was repeated section by section, from the deep to shallow regions until the planned target areas were ablated.

\section{Post-treatment follow-up}

After HIFU ablation, patients were carefully observed for 1-3 hours for possible complications before discharge. Oral antibiotics were prescribed for 7 days to prevent infection. Pelvic ultrasound (B-scan imaging, color Doppler flow imaging (CDFI), and power Doppler) was performed immediately after HIFU ablation to observe the therapeutic response. If a significant fraction of fibroids still had blood perfusion, additional HIFU ablation was performed immediately. The size of the ablated fibroid was measured in three orthogonal directions on ultrasound. The volume was simply calculated using the ellipsoid formula: (length $\times$ width $\times$ height $) \times 0.523(14)$.

\section{Statistics}

Data were initially summarized as mean \pm SD in a form of comparison tables and graphs. Statistical analysis was performed using the standard Statistical Package for the Social Sciences (SPSS Inc., Chicago, IL, USA) version 15 for windows. $P \leq 0.0001$ was considered to indicate a statistical significant difference. 


\section{RESULTS}

One hundred and fifty patients with single fibroids for each, were treated with HIFU. Among the 150 fibroids, 105 were located at the anterior wall of the uterus, 21 at the posterior wall of the uterus, 20 at the fundus, and 4 at the lateral wall. During HIFU ablation, all patients complained of a mild burning sensation in the abdominal skin, requiring no termination of treatment.

The treatment time ranged from 27 to 196 min (mean $85 \pm 43$ min). Details for the length of the procedure are as follows: room time, which including preparation time and treatment time, defined as the time from patient came with the HIFU unit to the patient went out the HIFU room, ranged from 3 to 8 hours.

The patients were continuously followed up until January 2015. Our results showed that the volumes began to decrease 1 month after HIFU ablation, and the regression of fibroids was obvious 3 months after HIFU ablation. At a median follow-up of 12 months, the ablated fibroids shrank significantly over time. The average volume shrinkage at $1,3,6$, and 12 months follow up was $(30.3 \%),(42.5 \%),(74.6 \%)$, and $(96.1 \%)$ on pelvic ultrasound, respectively. (Table 2 and Figure 1) describes the size change after HIFU ablation. During the follow- up period, a reduction in fibroid size was seen in $(150 ; 100 \%)$ fibroids; stable size was seen. 


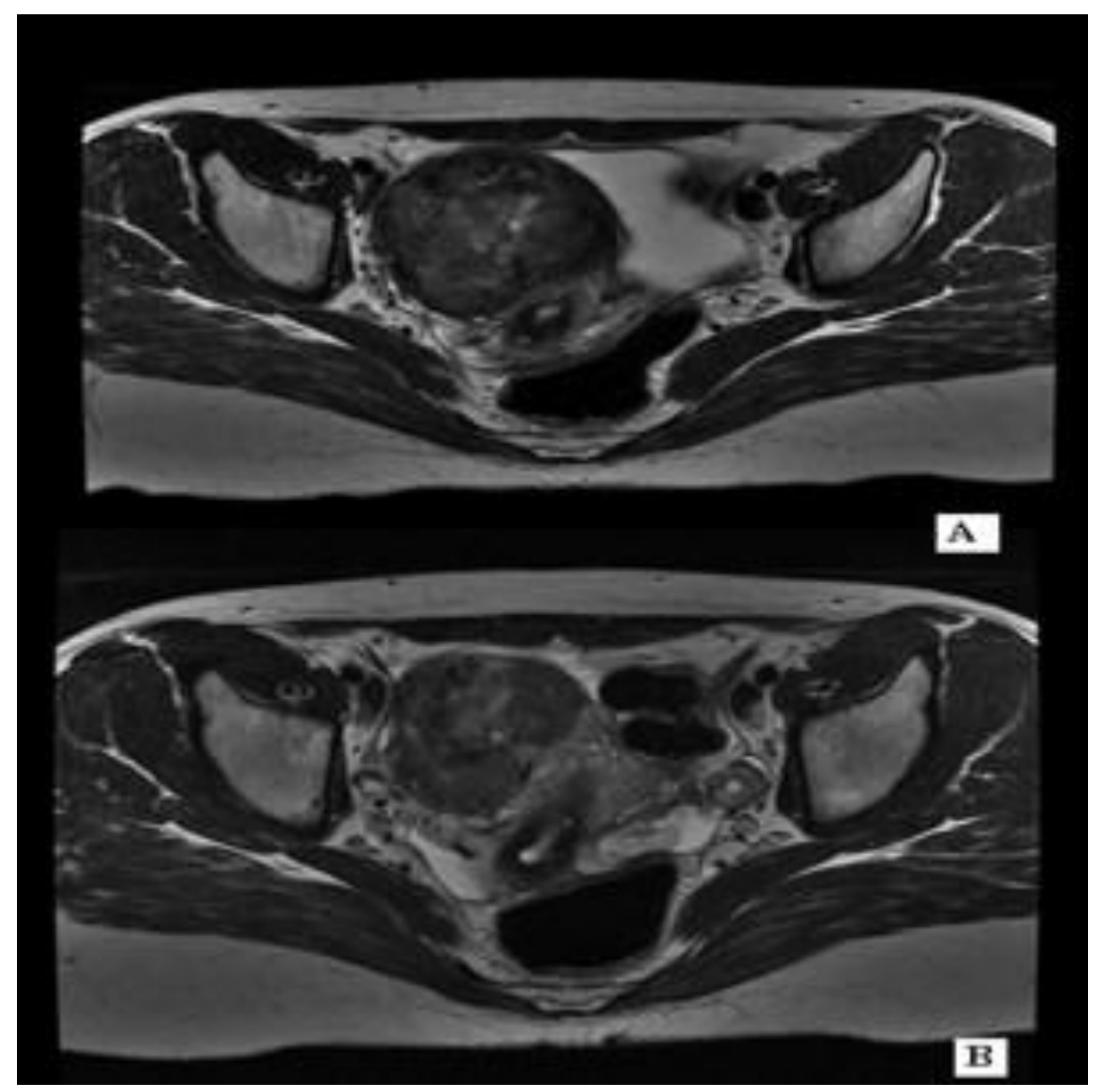

Figure 1. 25 years female with intramural fibroid. (A) Shows Axial $T_{2}$ FR-FSE (fast relaxation-fast spin echo) MRI image before HIFU fibroid treatment and (B) shows Axial $\mathrm{T}_{2}$ FR-FSE MRI image after 6 months of HIFU fibroid treatment. Shrinking in fibroid's size can be noticed after HIFU treatment.

All patients went home after 1-3 hours of observation, and administration of antibiotics was recommended for 7 days. Nurses called the patients daily during the first 3 days after HIFU treatment, but no one reported any clinically important symptoms. Major complications, which have previously led to substantial morbidity and disability corresponded to the unified standardized Society of Interventional Radiology (SIR) grading system (15) did not occur in this study. 
The volume of menstrual bleeding, decreased significantly $(P \leq 0.0001)$ the first menstrual period after HIFU ablation in $(65.4 \%)$ of patients. It also decreased significantly $(P$ $\leq 0.0001)$ in $(84.1 \%)$ and $(88.6 \%)$ of patients at 3 and 6 months after HIFU ablation. No patients had heavy menstrual bleeding, amenorrhoea, and anemia at 12 months after HIFU ablation. During the HIFU ablation, most patients felt minor abdominal pain, but as soon as the procedure stopped, the pain vanished. Among 150 patients treated with HIFU, an extremely low complication rate was observed (two patients $(1.3 \%)$ had low-grade fevers up to $38.1^{\circ} \mathrm{C}$ that persisted for approximately 1 to 3 days after HIFU ablation. (Table 3 ) lists complications occurring during and after HIFU ablation.

Expulsion of necrotic tissue from the vagina was observed in 12 patients. Intermittent mild pain was felt prior to the onset of vaginal discharge and was alleviated after vaginal discharge. The expulsion disappeared after 5-6 menstrual cycles. No patient reported leg or buttock pain after treatment. All patients returned to day-to-day activities one day after the procedure.

\section{DISCUSSION}

Because surgical treatments are associated with risks such as bleeding and infection, require a long recovery period and often necessitate general anaesthesia, several minimally invasive uterus preserving techniques have been developed for the treatment of symptomatic uterine fibroids, e.g. transarterial embolization (16,17), percutaneous ablation using cryoprobes (18), laser (19), and radiofrequency (20,21), and HIFU (22-24). Embolization can occlude the blood supply to the fibroids, resulting in tumor shrinkage and relief of symptoms, but it is associated with side effects like severe pelvic pain and complications such as premature menopause and ovarian failure. All percutaneous ablation techniques require insertion of 
needle-like applicators. It may be difficult to find a safe puncture route for placement of applicators in deeply located fibroids.

In this study, most of the uterine fibroids had a deep intramural extension and were considered difficult for complete resection (Table 1). However, ultrasound guided HIFU ablation yielded an encouraging therapeutic outcome. A high percentage of ablation necrosis was observed in all patients on pelvic ultrasound (Table 3). Unlike other minimally invasive techniques, HIFU ablation requires no insertion of applicators. The procedure can be easily repeated and large ablation areas can be induced under imaging guidance in one treatment session. These properties make it more advantageous for the treatment of uterine fibroids with deep intramural extension.

Further studies are warranted to compare HIFU ablation with other minimally invasive techniques in the treatment of uterine fibroids. Expulsion of necrotic fibroid tissue from the vagina was a common finding after ultrasound guided HIFU ablation. As it was associated with minor accompanying symptoms and required no medication, it was not regarded as a complication. Expulsion of uterine fibroids was also observed after uterine artery embolization (UAE) (25). The extent and length of vaginal expulsion after ultrasound guided HIFU ablation were similar to the results reported for transarterial embolization of submucosal fibroids (25). The expulsion of necrotic tissue may be a normal reaction after HIFU ablation of uterine fibroid. However, certain risk may still exist. If a large fibroid was expelled, it may obstruct the cervix and require hysteroscopic removal.

Safety is a major concern for HIFU ablation of symptomatic fibroids as the uterus is in close proximity to important structures, such as the bowel, bladder and lumbosacral plexus. To avoid thermal damage of these structures, the focus of the energy beam was kept at least 1 $\mathrm{cm}$ away from the tumor margin and the ablation area did not extend beyond the tumor margin. Irreversible thermal damage to the endometrium is a potential risk for HIFU ablation 
of submucosal fibroid which may cause amenorrhoea and infertility. Therefore, the authors only enrolled patients having no desire for future fertility. However, we tried to protect the endometrium during HIFU ablation in this study. The focus of the energy beam was kept at least $1 \mathrm{~cm}$ away from the endometrium. No patients had amenorrhoea after HIFU ablation in this study. The results suggested that by careful control of the focal region, HIFU ablation may not cause irreversible thermal damage to the endometrium, the endometrium may repair itself and patient's fertility may be preserved. Further studies are warranted to observe the influence of HIFU ablation on patient fertility.

In conclusion, the authors described their success with extracorporeal HIFU treatment of uterine fibroids. The authors report treatment efficacy based on imaging results. As confirmed in this study, imaging modalities are fairly insensitive in showing the necrotic area and residual disease after HIFU ablation. These findings validate HIFU ablation as a robust option for conservative treatment of uterine fibroids in patients who are unable to tolerate a hysterectomy and want to preserve their fertility. Nevertheless, large-scale randomized clinical trials are needed to determine the future role of this modality.

\section{ACKNOWLEDGEMENTS}

The authors extend their appreciation to the College of Applied Medical Sciences Research Center and the Deanship of Scientific Research at King Saud University for funding this research. The authors are really grateful to King Fahad Medical City (KFMC) to collaborate in this study. 


\section{REFERENCES:}

1. Phelps J. Uterine leiomyoma [abstract]. Environ Health Perspect 2005; 113: A740.

2. Carlson KJ, Nichols DH, Schiff I. Indications for hysterectomy. N Engl J Med 1993; 328: $856-860$.

3. Vollenhoven BJ, Lawrence AS, Healy DL. Uterine fibroids: a clinical review. Br J Obstet Gynaecol 1990; 97: 285-98.

4. Phelps J. Headliners: Uterine Leiomyoma: Genetic Reprogramming and Benign Uterine Tumors. Environ Health Perspect 2005; 113: A740.

5. Carlson KJ, Nichols DH, Schiff I. Indications for hysterectomy. N Engl J Med 1993; 328: $856-60$.

6. Wu F, Wang ZB, Chen WZ, Zou JZ, Bai J, Zhu H, et al. Extracorporeal focused ultrasound surgery for treatment of human solid carcinomas: early Chinese clinical experience. Ultrasound Med Biol 2004; 30: 245-60.

7. Smart OC, Hindley JT, Regan L, Gedroyc WM. Magnetic resonance guided focused ultrasound surgery of uterine fibroids-the tissue effects of GnRH agonist pre-treatment. Eur J Radiol 2006; 59: 163-67.

8. ter Haar G. High intensity ultrasound. Semin Laparosc Surg 2001; 8: 77-89.

9. Wu F, Wang ZB, Chen WZ, Zhu H, Bai J, Zou JZ, et al. Extracorporeal high intensity focused ultrasound ablation in the treatment of patients with large hepatocellular carcinoma. Ann Surg Oncol 2004; 11: 1061-69.

10. Häcker A, Köhrmann KU, Back W, Kraut O, Marlinghaus E, Alken P, et al. Extracorporeal application of high-intensity focused ultrasound for prostatic tissue ablation. BJU Int 2005; 96: 71-76. 
11. Yang R, Sanghvi NT, Rescorla FJ, Galliani CA, Fry FJ, Griffith SL, et al. Extracorporeal liver ablation using sonography-guided high-intensity focused ultrasound. Invest Radiol $1992 ; 27: 796-803$.

12. Kennedy JE. High-intensity focused ultrasound in the treatment of solid tumours. Nat Rev Cancer 2005; 5: 321-327.

13. Illing RO, Kennedy JE, Wu F, ter Haar GR, Protheroe AS, Friend PJ, et al. The safety and feasibility of extracorporeal high-intensity focused ultrasound (HIFU) for the treatment of liver and kidney tumours in a Western population. Br J Cancer 2005; 93: 890-895.

14. Kroencke TJ, Scheurig C, Kluner C, Taupitz M, Schnorr J, Hamm B. Uterine fibroids: contrast-enhanced MR angiography to predict ovarian artery supply --initial experience. Radiology 2006; 241: 181-189.

15. Goldberg SN, Grassi CJ, Cardella JF, Charboneau JW, Dodd III GD, Dupuy DE, et al. Image-guided tumour ablation: standardization of terminology and reporting criteria. Radiology 2005; 235: 728-39.

16. Bratby MJ, Hussain FF, Walker WJ. Outcomes after unilateral uterine artery embolization: a retrospective review. Cardiovasc Interv Radiol 2008; 31: 254-259.

17. Lumsden MA. Embolization versus myomectomy versus hysterectomy: which is best, when? Hum Reprod 2002; 17: 253-259.

18. Cowan BD, Sewell PE, Howard JC, Arriola RM, Robinette LG. Interventional magnetic resonance imaging cryoablation of uterine fibroids tumors: preliminary observation. Am J Obstet Gynecol 2002; 86: 1183-1187.

19. Hindley JT, Law PA, Hickey M, Smith SC, Lamping DL, Gedroyc WM, et al. Clinical outcomes following percutaneous magnetic resonance image guided laser ablation of symptomatic uterine fibroids. Hum Reprod 2002; 17: 2737-41. 
20. Carrafiello G, Recaldini C, Fontana F, Ghezzi F, Cuffari S, Laganà D, et al. Ultrasoundguided radiofrequency thermal ablation of uterine fibroids: medium-term follow-up. Cardiovasc Intervent Radiol 2010; 33: 113-119.

21. Recaldini C, Carrafiello G, Laganà D et al. Cuffari S, Bergamini V, Ghezzi F Percutaneous sonographically guided radiofrequency ablation of medium-sized fibroids: feasibility study. AJR Am J Roentgenol 2007; 189: 1303-1306.

22. Ren XL, Zhou XD, Zhang J, He GB, Han ZH, Zheng MJ, et al. Extracorporeal ablation of uterine fibroids with high-intensity focused ultrasound: imaging and histopathologic evaluation. J Ultrasound Med 2007; 26: 201-212.

23. LeBlang SD, Hoctor K, Steinberg FL. Leiomyoma shrinkage after MRI-guided focused ultrasound treatment: report of 80 patients. AJR Am J Roentgenol 2010; 194: 274-280.

24. Chapman A, ter Harr G. Thermal ablation of uterine fibroids using MR-guided focused ultrasound-a truly non-invasive treatment modality. Eur Radiol 2007; 17: 2505-11.

25. Radeleff B, Eiers M, Bellemann N, Ramsauer S, Rimbach S, Kauczor HU, et al. Expulsion of dominant submucosal fibroids after uterine artery emobolization. Eur J Radiol 2010; 75: e57-e63. 
TABLES:

Table 1. Scanning parameters for the routine MDCTU protocol.

$$
\text { Age (years) }
$$

\begin{tabular}{cc} 
Mean \pm SD & $38.1 \pm 1.3$ years \\
Range & $28-48$ years \\
\hline Married & Martial Status, n (\%) \\
Divorced & $79 ; 52.7 \%$ \\
Single & $32 ; 21.3 \%$ \\
\hline Anemia & $39 ; 26 \%$ \\
\hline Urinary tract symptoms & Symptoms, n (\%) \\
Dysmenorrhoea & $15 ; 10 \%$ \\
Menorrhagia & $18 ; 12 \%$ \\
\hline
\end{tabular}

Fibroid location

\begin{tabular}{ccc}
\hline Subserosal & $27 ; 18 \%$ \\
Submucosal & $59 ; 39.3 \%$ \\
Intramural & $64 ; 42.7 \%$ \\
\hline & Fibroid diameter, cm & \\
\hline Mean \pm SD & & $5.2 \pm 1.8 \mathrm{~cm}$ \\
Range & $3.9-10.6 \mathrm{~cm}$ \\
\hline Mean \pm SD & Fibroid volume, $\mathrm{cm}^{3}$ & \\
\hline Range & & $75.2 \pm 1.6 \mathrm{~cm}^{3}$ \\
\hline
\end{tabular}


Table 2. Follow-up size change after HIFU ablation.

\begin{tabular}{cccc}
\hline Follow-up time & Diameter, $\mathrm{cm}$ & Volume, $\mathrm{cm}^{3}$ & Volume shrinkage (\%) \\
\hline Pre-treatment & $5.2 \pm 1.8 \mathrm{~cm}$ & $45.2 \pm 1.6 \mathrm{~cm}^{3}$ & - \\
1 month post-treatment & $3.6 \pm 1.8 \mathrm{~cm}$ & $31.5 \pm 1.6 \mathrm{~cm}^{3}$ & $30.3 \%$ \\
3 months post-treatment & $2.99 \pm 1.8 \mathrm{~cm}$ & $26 \pm 1.6 \mathrm{~cm}^{3}$ & $42.5 \%$ \\
6 months post-treatment & $1.3 \pm 1.8 \mathrm{~cm}$ & $11.5 \pm 1.6 \mathrm{~cm}^{3}$ & $74.6 \%$ \\
12 months post-treatment & $0.2 \pm 1.8 \mathrm{~cm}$ & $1.8 \pm 1.6 \mathrm{~cm}^{3}$ & $96.1 \%$ \\
\hline
\end{tabular}


Table 3. Complications occurring during and after HIFU ablation.

\begin{tabular}{ccc}
\hline Symptoms and signs & Duration, day & Patients, $\mathrm{n}(\%)$ \\
Low grade fever & $1-3$ day & $2 ; 1.3 \%$ \\
Hematuria & $2-4$ day & $7 ; 4.7 \%$ \\
Lower abdominal pain & $1-3$ day & $4 ; 2.7 \%$ \\
Vaginal bleeding & $2-5$ day & $4 ; 2.7 \%$ \\
Vaginal discharge & $4-8$ day & $12 ; 8 \%$ \\
No specific symptoms & - & $65 ; 43.3 \%$ \\
\hline
\end{tabular}

The effectiveness of HIFU treatment for ablating of uterine fibroids

${ }^{1}$ Department of Radiological Sciences, College of Applied Medical Sciences, King Saud University, Riyadh, Saudi Arabia

${ }^{2}$ Radiology and Medical Imaging Department, College of Applied Medical Sciences, Salman bin Abdulaziz University, Al-Kharj, Saudi Arabia

${ }^{3}$ Basic Sciences Department, College of Medical Radiological Sciences, Sudan University of Science and Technology, Khartoum, Sudan 
${ }^{4}$ Department of Diagnostic Radiology, American University of Beirut Medical Center, Beirut, Lebanon

${ }^{5}$ Radiation Therapy Department, King Fahad Medical City, Riyadh, Saudi Arabia

*Corresponding author: Dr. Mustafa Z. Mahmoud, Radiology and Medical Imaging Department, College of Applied Medical Sciences, Salman bin Abdulaziz University, PO Box 422, Al-Kharj 11942, Saudi Arabia; Tel: 00966115443928; Fax: 00966115886301; Email: $\underline{\text { m.alhassen@sau.edu.sa }}$

Synopsis: Authors aim in this prospective cohort study to evaluate the effectiveness of high intensity focused ultrasound (HIFU) ablation for the treatment of uterine fibroids. 